\title{
Experimental evidence of coexistence of proper magnetic and structural incommensurability on $\left[\mathrm{CH}_{3} \mathrm{NH}_{3}\right]\left[\mathrm{Ni}(\mathrm{COOH})_{3}\right]$ compound
}

Laura Cañadillas-Delgado, ${ }^{1 *}$ Lidia Mazzuca, ${ }^{1}$ Oscar Fabelo, ${ }^{1 *}$ Juan RodríguezCarvajal, ${ }^{1}$ Vaclav Petricek ${ }^{2}$

${ }^{1}$ Institut Laue-Langevin, 71 Avenue des Martyrs, CS 20156, 38042 Grenoble Cedex 9, France.

${ }^{2}$ Institute of Physics, Academy of Sciences of the Czech Republic, Na Slovance 2, 18040 Praha 8 , Czech Republic.

Corresponding Authors: lcd@ill.fr,fabelo@ill.fr

Supplementary Information

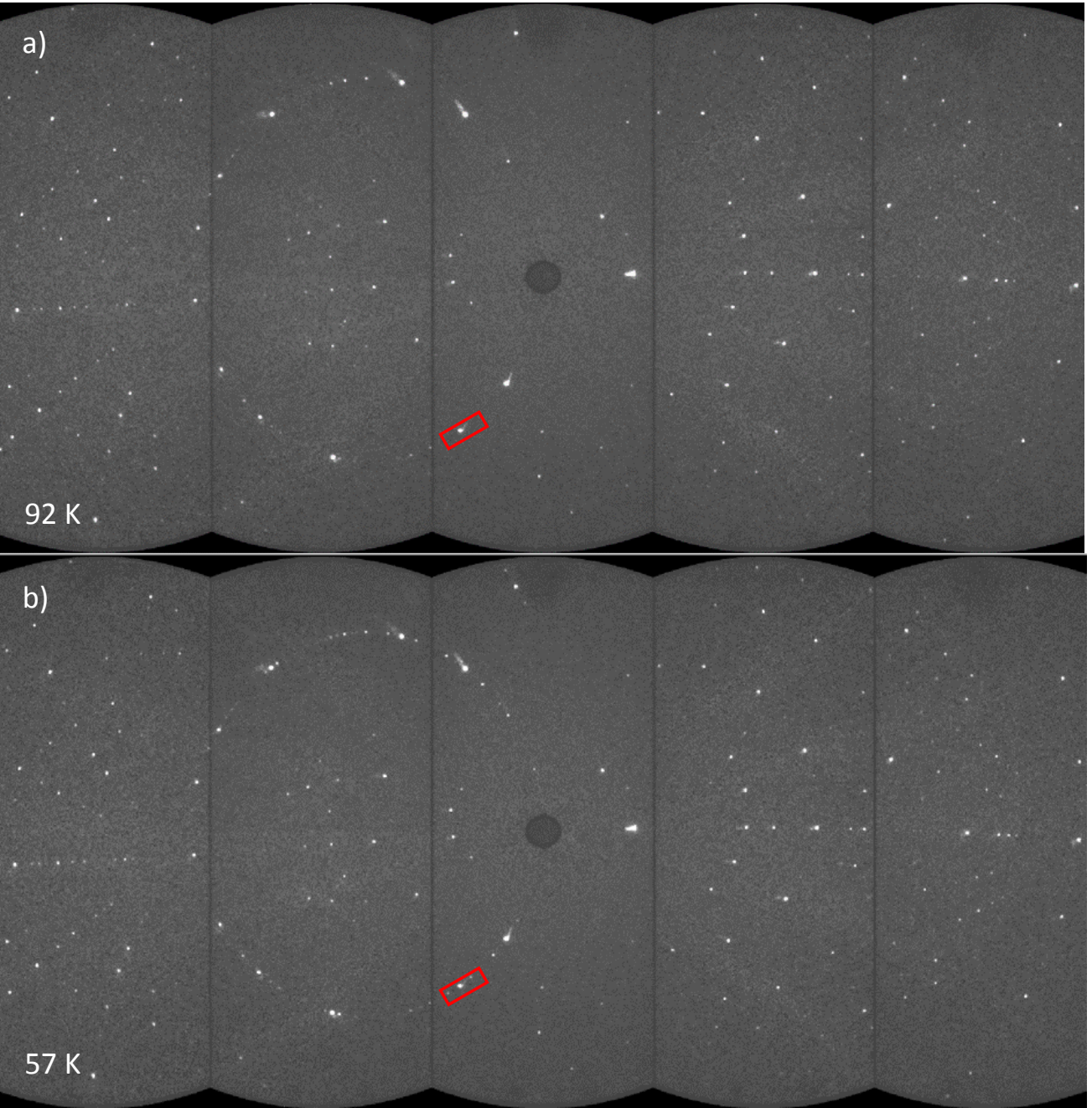


c)

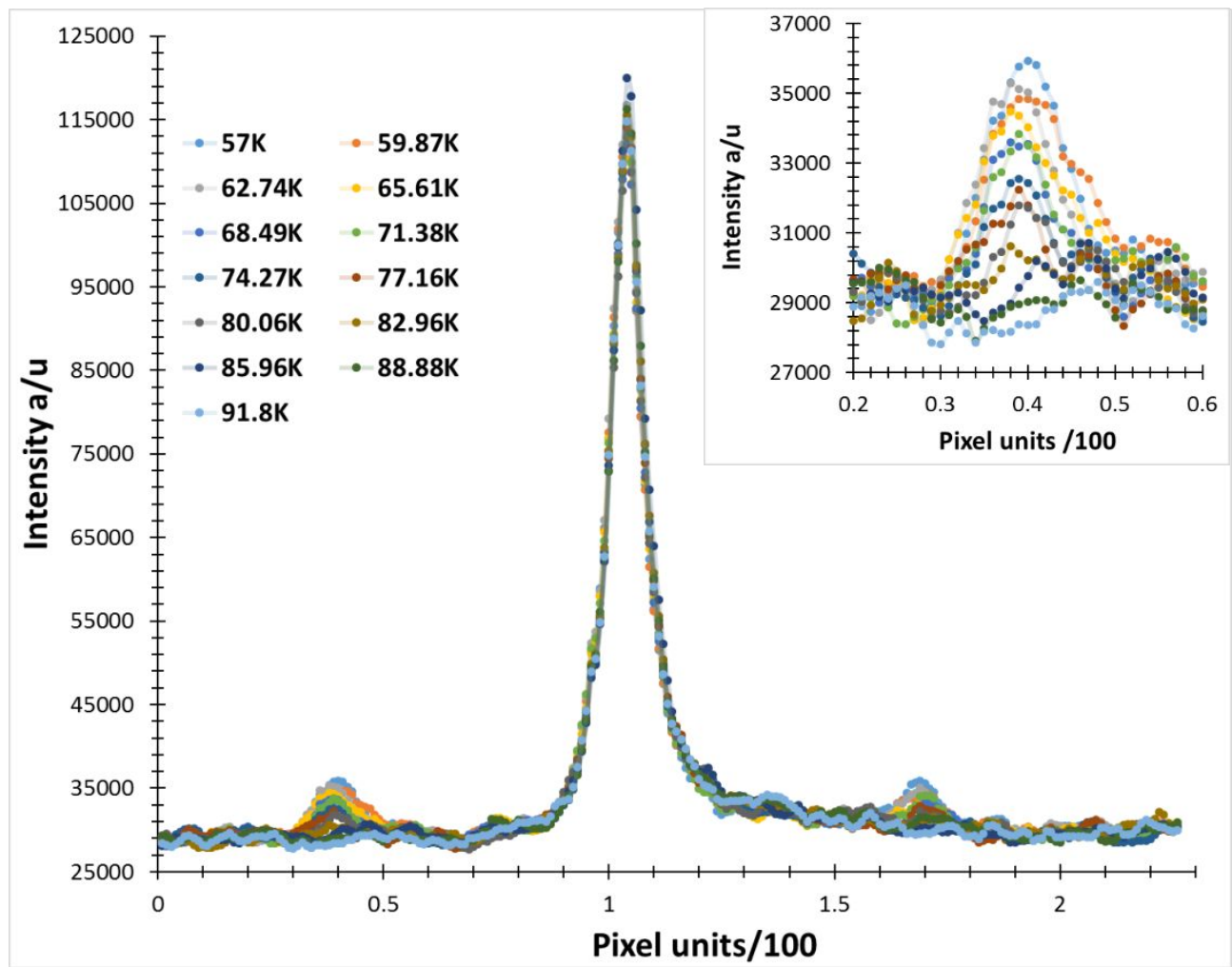

Figure S1. Temperature evolution of the neutron Laue patterns. (a) Pattern collected at $92 \mathrm{~K}$ corresponding to the orthorhombic phase in the Pnma space group. (b) Pattern collected at $57 \mathrm{~K}$ corresponding to the incommensurate phase in the Pnma $(00 \gamma) 0 s 0$ super space group. (c) Evolution in temperature of the intensity of the highlighted areas in (a) and (b), where it can be appreciated the appearance of satellite reflections at ca. $84 \mathrm{~K}$. Inset: Detail of the intensity of one of the satellite reflections in the zoomed area of (a) and (b). 


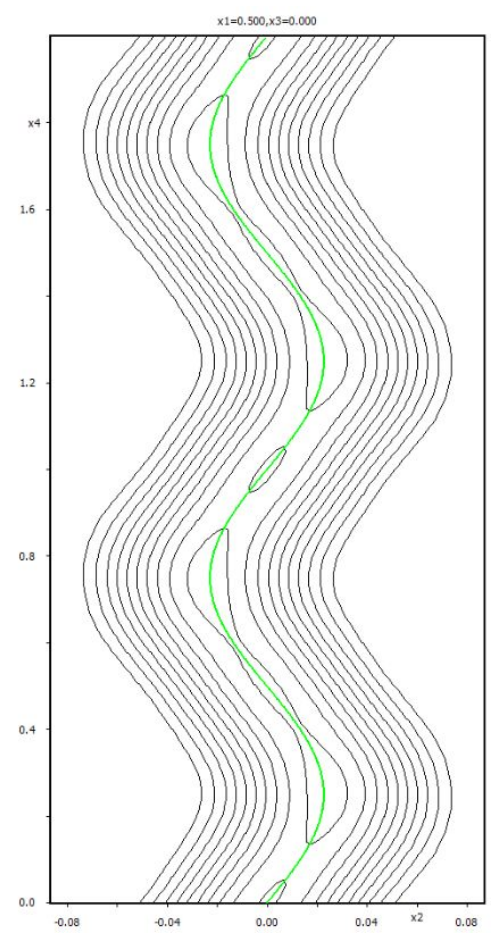

Figure S2. Representation of the refined modulation functions for the nickel atom (green) at 40(1) $\mathrm{K}$, which is in good agreement with the experimental $\left(\mathrm{F}_{\mathrm{obs}}\right)$ Fourier maps. The contour plot of the $\mathrm{x}_{4}-\mathrm{x}_{2}$ two-dimensional sections have been calculated fixing $\mathrm{x}_{1}=0.5$ and $\mathrm{x}_{3}=0$, corresponding to the atomic position of the nickel atom.

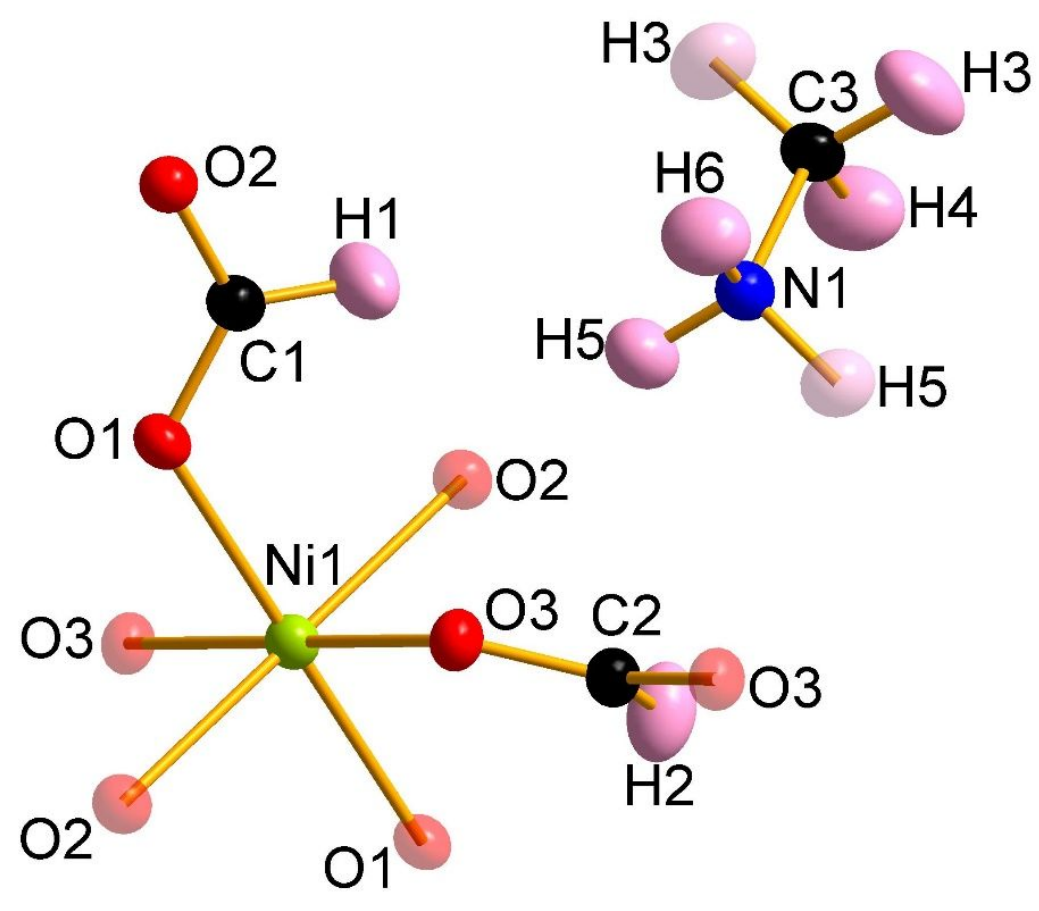


Figure S3. View of the asymmetric unit of the average structure of compound 1 at 40(1) K, represented at $50 \%$ of probability, obtained from neutron data. The atoms generated by symmetry have been represented with transparency. Nickel, oxygen, nitrogen, carbon and hydrogen atoms have been represented in green, red, blue, black and pink colours, respectively.

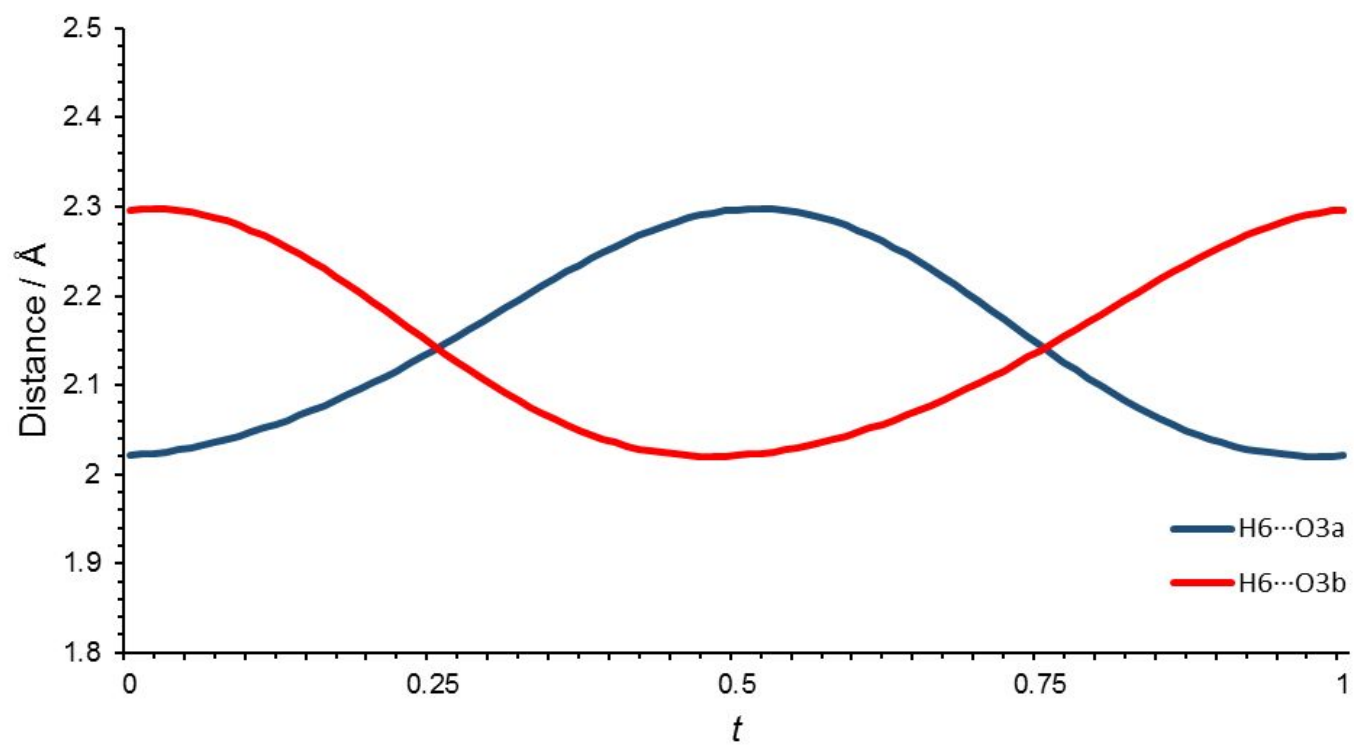

Figure S4. Modulation of the bond lengths between the third hydrogen atom of the $\mathrm{NH}_{3}$ group of the methylammonium counter-ion and the closest oxygen atom from formate ligand at 40(1) $\mathrm{K}$. Symmetry codes: $a=x+1 / 2,-y+1 / 2,-z-1 / 2 ; b=x+1 / 2, y,-z-1 / 2$

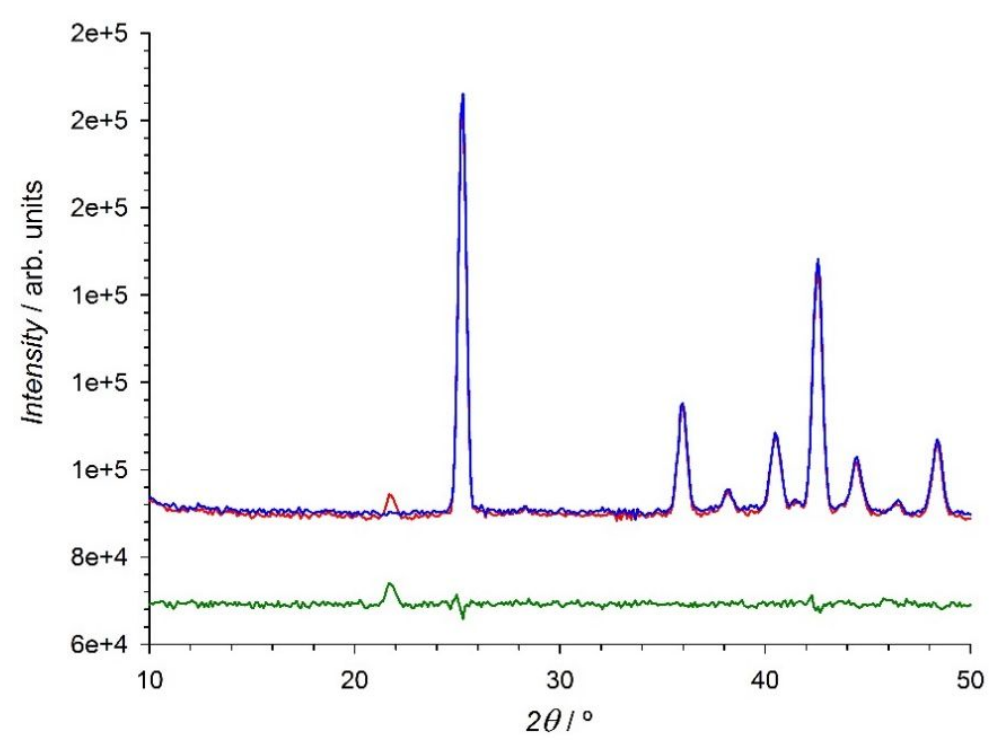

Figure S5. Neutron powder patterns of compound 1 collected in the ordered phase at $2 \mathrm{~K}$ (red), and in the paramagnetic phases, at $40 \mathrm{~K}$ (blue). The difference pattern has been represented as a solid green line. 
Table S1. Selected distances $(\AA)$ and angles $\left(^{\circ}\right)$ involving the methylammonium counter-ion at 40(1) K. Distances and angles further from the ideal hydrogen-bond geometry are emphasized in italics. Symmetry codes: $a=x+1 / 2,-y+1 / 2,-z-1 / 2 ; b=x+1 / 2, y,-z-1 / 2 ; c=-x+3 / 2,-y, z+1 / 2$

\begin{tabular}{l|l|l|l|l|l|l|l|l|l} 
& \multicolumn{1}{l}{$\mathrm{N} 1-\mathrm{H} 6 \cdots \mathrm{O} 3 \mathrm{a}$} & $\mathrm{N} 1-\mathrm{H} 6 \cdots \mathrm{O} b \mathrm{~b}$ & \multicolumn{2}{l}{$\mathrm{N} 1-\mathrm{H} 5 \cdots \mathrm{O} 2 \mathrm{c}$} \\
\hline $\mathrm{t}$ & $\mathrm{H} \cdots \mathrm{A}$ & $\mathrm{D} \cdots \mathrm{A}$ & $\mathrm{D}-\mathrm{H} \cdots \mathrm{A}$ & $\mathrm{H} \cdots \mathrm{A}$ & $\mathrm{D} \cdots \mathrm{A}$ & $\mathrm{D}-\mathrm{H} \cdots \mathrm{A}$ & $\mathrm{H} \cdots \mathrm{A}$ & $\mathrm{D} \cdots \mathrm{A}$ & $\mathrm{D}-\mathrm{H} \cdots \mathrm{A}$ \\
\hline 0 & $2.021(9)$ & $2.972(4)$ & $154.0(6)$ & $2.297(8)$ & $3.115(4)$ & $136.2(4)$ & $1.841(8)$ & $2.867(2)$ & $174.2(7)$ \\
\hline 0.1 & $2.047(9)$ & $2.980(4)$ & $152.1(6)$ & $2.274(8)$ & $3.102(4)$ & $138.0(4)$ & $1.831(8)$ & $2.868(2)$ & $174.9(7)$ \\
\hline 0.2 & $2.102(9)$ & $3.014(4)$ & $147.1(5)$ & $2.194(8)$ & $3.067(4)$ & $142.1(5)$ & $1.820(8)$ & $2.862(2)$ & $175.0(7)$ \\
\hline 0.3 & $2.179(8)$ & $3.060(4)$ & $141.4(5)$ & $2.099(8)$ & $3.025(4)$ & $147.4(5)$ & $1.815(8)$ & $2.853(2)$ & $174.0(7)$ \\
\hline 0.4 & $2.256(8)$ & $3.099(4)$ & $137.4(4)$ & $2.035(9)$ & $2.990(4)$ & $152.0(6)$ & $1.812(8)$ & $2.843(2)$ & $172.0(7)$ \\
\hline 0.5 & $2.297(8)$ & $3.115(4)$ & $136.2(4)$ & $2.021(9)$ & $2.972(4)$ & $154.0(6)$ & $1.808(8)$ & $2.836(2)$ & $170.9(7)$ \\
\hline 0.6 & $2.274(8)$ & $3.102(4)$ & $138.0(4)$ & $2.047(9)$ & $2.980(4)$ & $152.1(6)$ & $1.803(8)$ & $2.835(2)$ & $172.2(7)$ \\
\hline 0.7 & $2.194(8)$ & $3.067(4)$ & $142.1(5)$ & $2.102(9)$ & $3.014(4)$ & $147.1(5)$ & $1.807(8)$ & $2.841(2)$ & $174.5(7)$ \\
\hline 0.8 & $2.099(8)$ & $3.025(4)$ & $147.4(5)$ & $2.179(8)$ & $3.060(4)$ & $141.4(5)$ & $1.822(8)$ & $2.850(2)$ & $174.5(7)$ \\
\hline 0.9 & $2.035(9)$ & $2.990(4)$ & $152.0(6)$ & $2.256(8)$ & $3.099(4)$ & $137.4(4)$ & $1.838(8)$ & $2.861(2)$ & $173.9(7)$ \\
\hline 1.0 & $2.021(9)$ & $2.972(4)$ & $154.0(6)$ & $2.297(8)$ & $3.115(4)$ & $136.2(4)$ & $1.841(8)$ & $2.867(2)$ & $174.2(7)$ \\
\hline
\end{tabular}

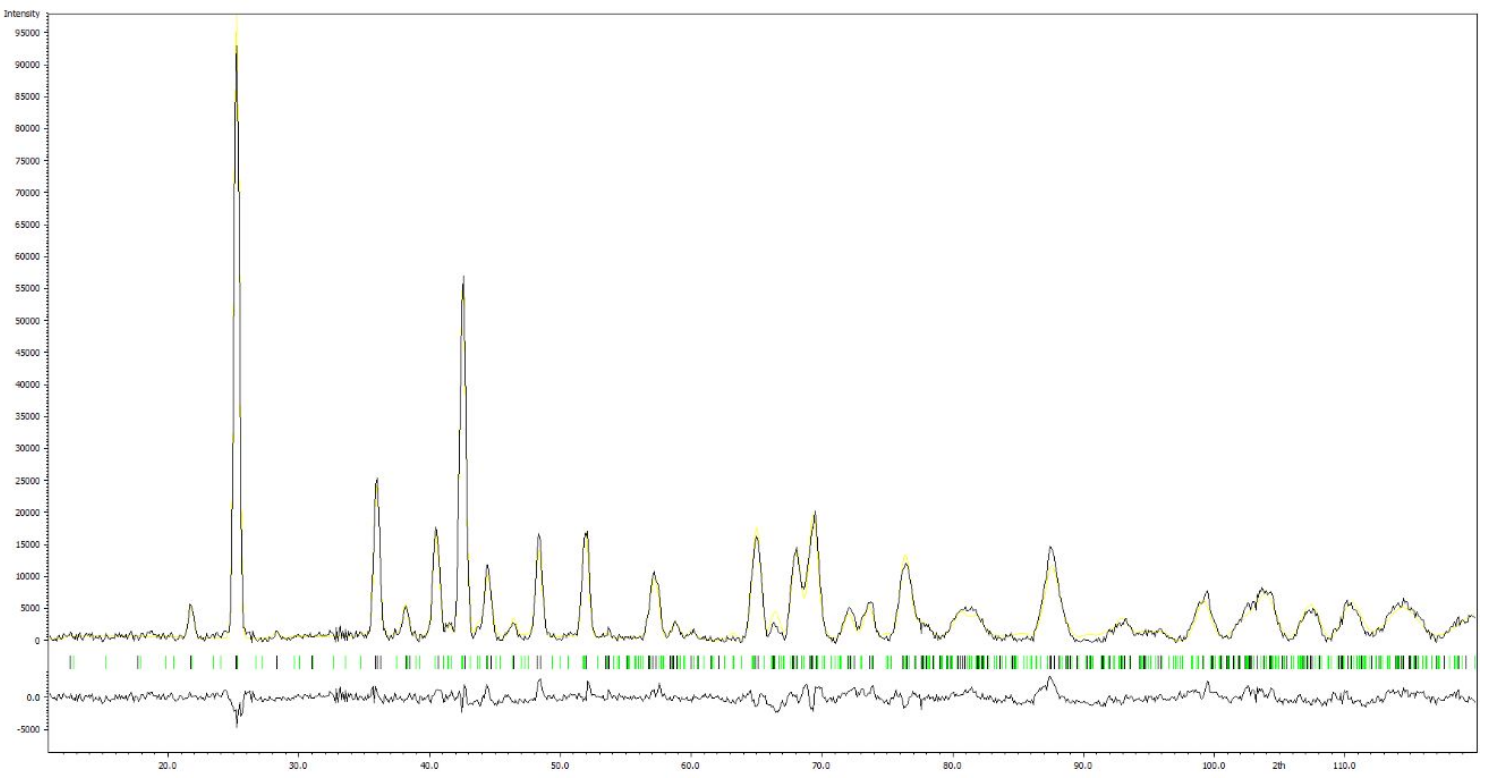

Figure S6. Fit of the neutron powder pattern at $2 \mathrm{~K}$ using the $P n^{\prime} m a^{\prime}(00 \gamma) 0 s 0$ super-space group. For the sake of clarity, the background contribution has been removed. The vertical lines corresponds with the allowed reflections $(\mathrm{hklm})$. 
Table S2. Results of refinement of the magnetic model of compound 1 at 5(1) K. The different refinements corresponds with: (a) the magnetic model without considering magnetic modulation, thus only structural displacive modes have been taken into account; (b) the magnetic model considering magnetic modulations; (c) the magnetic model considering magnetic modulations but with restricted value of the magnetic moment modulus.

\begin{tabular}{llll}
\hline Superspace group & \multicolumn{2}{c}{$P n^{\prime} m a^{\prime}(00 \gamma) 0 s 0$} \\
Type of refinement & (a) & (b)* & (c) \\
$\mathrm{R}_{\text {obs, }} \mathrm{I}>2 \mathrm{~s}(\mathrm{I})$ & 7.85 & 7.73 & 7.78 \\
$\mathrm{WR}_{\mathrm{obs}}, \mathrm{I}>2 \mathrm{~s}(\mathrm{I})$ & 16.99 & 16.32 & 16.64 \\
$\mathrm{R}_{\text {all }}$ & 11.28 & 11.09 & 11.19 \\
$\mathrm{WR}_{\text {all }}$ & 18.16 & 17.37 & 17.75 \\
Magnetic moment $/ \mu_{\mathrm{B}}$ & $2.13(12)$ & $1.77(12)-2.63(12)$ & $2.14(9)$
\end{tabular}

* The final magnetic moments of some sites have no-physical meaning and therefore this model can be discarded.

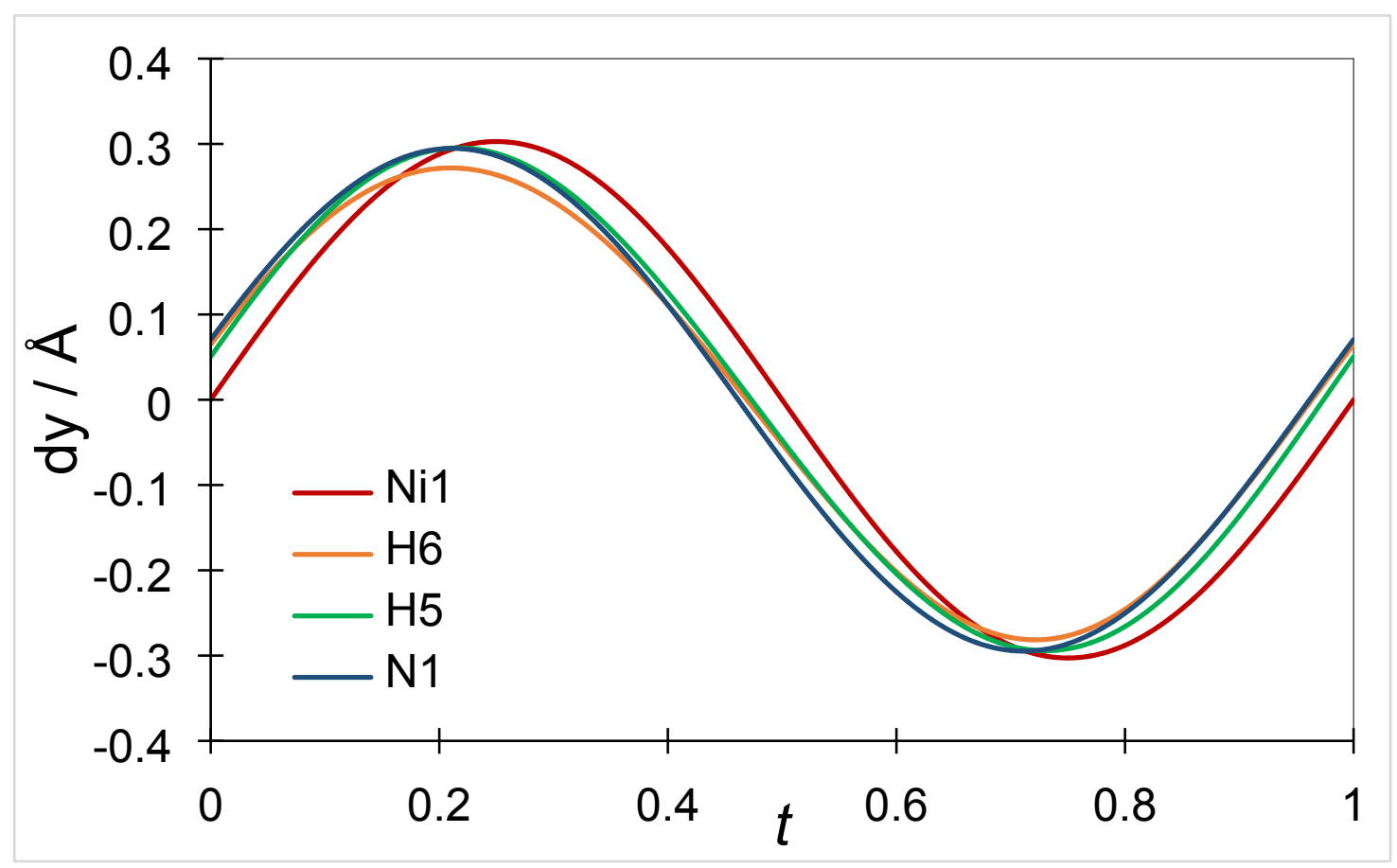

Figure S7. Comparison of the modulation displacements for selected atoms along the $b$-axis. 
